MATHEMATICAL ASSOCIATION

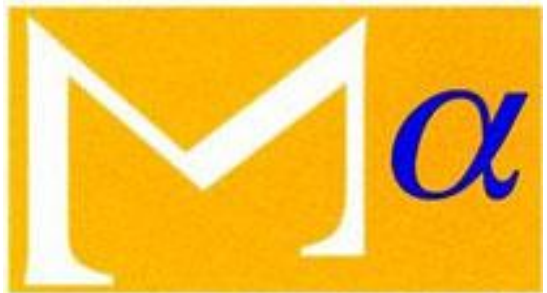

supporting mathematics in education

The Teaching of Easy Calculus to Boys (Continued)

Author(s): W. Knowles

Source: The Mathematical Gazette, Vol. 7, No. 111 (May, 1914), pp. 322-325

Published by: Mathematical Association

Stable URL: http://www.jstor.org/stable/3602668

Accessed: 18-01-2016 17:01 UTC

Your use of the JSTOR archive indicates your acceptance of the Terms \& Conditions of Use, available at http://www.jstor.org/page/ info/about/policies/terms.jsp

JSTOR is a not-for-profit service that helps scholars, researchers, and students discover, use, and build upon a wide range of content in a trusted digital archive. We use information technology and tools to increase productivity and facilitate new forms of scholarship. For more information about JSTOR, please contact support@jstor.org. 
obscurity-how easily a teacher may aim too high, and in striving after mathematical elegance, precision, and generality become unintelligiblehow easily a teacher may aim too low and sink to the mean and dull through anxiety to keep well within the comprehension of his pupils-that our task of trying to teach people to think by showing them methods of avoiding laborious thinking is not a very simple one, that it is wrong always to puzzle your pupils and also wrong never to puzzle them. Therefore, though some dogmatic statements have been made, I would end by disclaiming any intention to lay down as law any proposition save one-that this problem must be approached in a broad-minded way. C. S. JACkson.

\section{THE TEACHING OF EASY CALCULUS TO BOYS.}

\section{(Continued from p. 208.)}

ANother useful application is that the parabola, in the form $y=-k \cdot x^{2}$, represents the path of a projectile. The boys at this stage have already dealt with equations of the form $y= \pm(x-a)(x-b)$, and are able to find the points where the curve is cut by the $x$ or the $y$-axis.

Hence, in the equation $y=-(t-a)(t-b)$, since $y$ denotes the height of a point on the curve above the $x$-axis, $\frac{d y}{d t}$ gives the vertical velocity at time $t$.

The velocity curve $\frac{d y}{d t}$ against $t$ is drawn, and the question arises, when is the velocity zero? or where is the highest point of the curve? and we enter upon the subject of Maximum and Minimum Values.

This is first discussed as finding the highest or the lowest point of a given parabola. A parabola such as $2 y=-(x+2)(x-3)$ is drawn, and several successive values of $\frac{\delta y}{\delta x}$ are found and compared. It is thus shown that $\delta y$ $\delta y$ gets smaller and smaller until at a certain point $\delta y=0$, after which it becomes negative.

Plenty of illustrations will occur here (e.g. a man walking over a hill top) to show that at the highest point of the curve the direction is for an instant horizontal. A reference to the mountain railway at the White City never fails to arouse interest, and the boys soon appreciate what the change in $\frac{d y}{d x}$ means, and there is little difficulty in getting them to see that when they are at the top of the hill, then $\frac{d y}{d x}=0$.

(The other case-the minimum point-is reserved for a subsequent lesson.)

Exercises are then worked. Find the coordinates of the highest point of the curve of which the general equation is

$$
y=a+b x+c x^{2} \text {. }
$$

When this is fairly well grasped a parabola of the form $y=+k x^{2}$ is taken, and by following similar lines to those in the previous discussion, the minimum point is found, and some exercises of this type are worked.

Then comes the question: Since the equation $\frac{d y}{d x}=0$ gives both the highest as well as the lowest points of the curves, and the process of working is similar in each case, how shall we distinguish between then without the trouble of graphing the curve. Here again the car on the mountain railway will carry us over these points. What is the slope of the car when

$$
\frac{d y}{d x} \text { is }+, \text { when } \frac{d y}{d x} \text { is } 0 \text {, and when } \frac{d y}{d x} \text { is - ? }
$$


and we arrive at the rule that in passing over a maximum point the order of change in the value of $\frac{d y}{d x}$ is $+, 0,-$, while in passing a minimum point the order of change is $-, 0,+$.

Hence we find, by trial, the value of $\frac{d y}{d x}$ when $x$ is a little less than, and again when $x$ is a little more than the value which makes $\frac{d y}{d x}=0$.

Note.-I have not found the method of taking the second differential very successful with boys. There seems to be a lack of suitable illustrations.

This subject opens up a wide field for exercises of a problem kind, and which can be given at frequent intervals throughout the remainder of the course. In these problems the real difficulty is to get the boys to state the conditions as a mathematical equation.

The next figure that we study is the circle.

The same method of investigation that has been adopted for the parabola is followed here. This being taken for granted, it will not be necessary for me now to dwell upon those parts which are similar to the preceding work, but it will be sufficient to show how new methods are introduced, aud any other points of special interest.

As before, new mathematical operations are taught as the need for them arises.

The boys will already know the equation of the circle, and a first exercise is to find the value of $\frac{\delta y}{\delta x}$. This is done by taking two points,

$$
\left(x-\delta x_{1}\right),\left(y-\delta y_{1}\right) \text { and }\left(x+\delta x_{1}\right),\left(y+\delta y_{2}\right),
$$

one on each side of the point $(x, y)$ on the circle, and we have

$$
\begin{aligned}
& \left(y-\delta y_{1}\right)^{2}=r^{2}-\left(x-\delta x_{1}\right)^{2}, \\
& \left(y+\delta y_{2}\right)^{2}=r^{2}-\left(x+\delta x_{1}\right)^{2},
\end{aligned}
$$

from which we get

$$
\frac{\delta y}{\delta x}=-\frac{x}{y} \text {. }
$$

This result is verified for various points on the circumference.

(2) The value of $\frac{d y}{d x}$ may be also found by the method of substitution, i.e. by letting $y^{2}=z$; in fact, this problem becomes the peg upon which to hang this method of substitution.

No particular difficulty has been found in getting boys to grasp this method. First find $\frac{d y}{d z}$, then $\frac{d z}{d x} ; d z$ is the connecting link. Plenty of examples for practice should be worked.

The area of a circle is found by assuming the circle to be divided into a large number of sectors. The method by division into strips parallel to the $y$-axis may be deferred for the present if they are not sufficiently advanced with their Trigonometry.

It is well now to take the volume of the sphere. Consider the hemisphere to be generated by the rotation of a quadrant of the circle about the $x$-axis, and we have

$$
\text { the volume of hemisphere }=\pi \int_{0}^{\frac{\pi}{2}} y^{2} d x,
$$

and by varying the limits the volume of a section of the sphere is obtained.

The next investigation is to study by what law the value of the sine of an 
angle changes as the angle itself changes, that is, to find the value of the ratio $\frac{\delta \sin \theta}{\delta \theta}$ for any given value of $\theta$.

The boys will be now quite familiar with the measurement of angles in radians.

(1) The first method is the usual geometrical method, from which it is made clear that the value of $\frac{\delta \sin \theta}{\delta \theta}$ approximates to $\cos \theta$ as $\delta \theta$ dinimishes.

(2) The second method is as follows :

Let

$$
\begin{gathered}
y-\delta y_{1}=\sin \left(\theta-\delta \theta_{1}\right), \\
y+\delta y_{1}=\sin \left(\theta+\delta \theta_{1}\right), \\
\delta y=\cos \theta\left(\frac{\sin \delta \theta_{1}}{\delta \theta_{1}}\right) .
\end{gathered}
$$

and we obtain

Here $\frac{\sin \delta \theta_{1}}{\delta \theta_{1}}$ is the factor causing an error due to the size of $\delta \theta$.

When $\delta \theta_{1}=\frac{\pi}{22}$, then $\frac{\sin \delta \theta_{1}}{\delta \theta_{1}}=.997$ approximately, and $\therefore 7 \delta y=\cdot 997 \cos \theta$.

This equation provides us with a good practical method of differentiating graphically the sine curve.

Take $\delta \theta=\frac{\pi}{22}$, i.e. divide $\pi$ radians on the $x$-axis into 22 equal parts. Then multiply each $\delta y$ by 7 , and the derived curve is very nearly of the same amplitude as the original sine curve.

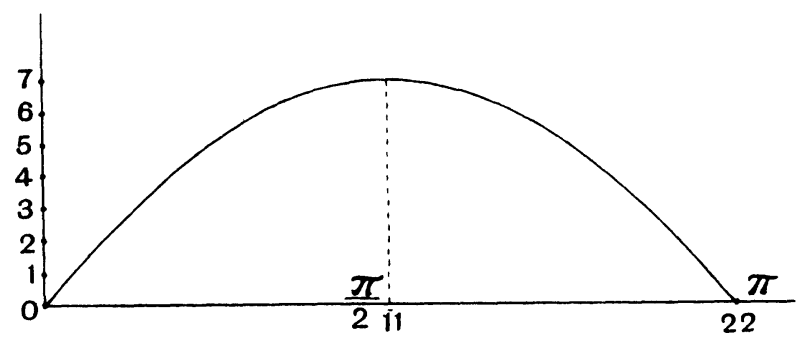

The value of $\frac{\delta \cos \theta}{\delta \theta}$ is obtained by similar methods.

Each of these results is verified numerically with the help of tables.

The reverse exercises are then worked as graphic integration, and the boys should have little difficulty in finding out the method unaided.

Exercises follow upon the area of the sine curve between given limits.

The next step is to differentiate the forms :

and

$$
\begin{aligned}
& y=A \sin \theta, \\
& y=\sin (n \theta), \\
& y=\sin (\theta+\alpha), \\
& y=A \sin \{(n \theta)+\alpha\} .
\end{aligned}
$$

By this time a greater variety of exercises is possible, many of which can be set in problem form.

The area of a circle in the form

$$
\text { Area }=\int_{0}^{r} \sqrt{r^{2}-x^{2}} d x
$$


may be now found by changing the integral to the form

$$
r^{2} \int_{0}^{\frac{\pi}{2}} \cos ^{2} \theta d \theta
$$

and this serves as an introduction to integration by parts and the differentiation of a product; but this part may be left to a later stage, or in some classes is omitted altogether.

At somewhere about this stage of the work exercises are brought in on calculating the value of the sine and cosine of a given angle, and also calculating the value of $\pi$.

The boys have been using for some time tables of sines, and they are really interested in the question-How were these tables first got out? and they are keen on being able to calculate the value of sine $\frac{1}{2}$ radian for themselves without any help from books.

The method is on these lines. They are reminded that

$$
\begin{aligned}
& y=a+b x \text { represents a straight line ; } \\
& y=a+b x+c x^{2} \text { represents a parabola; } \\
& y=a+b x+c x^{2}+d x^{3} \text { represents a cubic curve, and so on ; }
\end{aligned}
$$

and then I tell them that it has been proved that certain curves can be represented by equations of this form ; that in some cases a very large number of terms must be taken; that the subject is dealt with in books on the Calculus, under Taylor's theorem, and that $y=\sin \theta$ is one of these curves. Then

$$
\sin \theta \text { is assumed }=a+b \theta+c \theta^{2}+d \theta^{3} \text {, etc., }
$$

and by alternately making $\theta=0$, and, by differentiating, the values of $a, b, c$, $d$, etc., are obtained.

Then by taking $\theta=.5$ in the series obtained, the value of $\sin \cdot 5$ radian is obtained.

The series for $\cos \theta$ is worked out in the same way; the value of $\frac{\pi}{4}$ has also been calculated on similar lines.

The boys need a fair amount of help to get through these the first time, then the formal workings are entered in their note books for reference, and these provide material for further exercises in calculation.

In dealing with logarithms and the hyperbola, the use of the Calculus plays only a small, although an important part of the whole work, and time will permit me to indicate only the nature of the work done. The curve $y=e^{x}$ is differentiated both graphically and algebraically, and the nature of the error due to the finite size of $\delta x$ is pointed out.

The curve $y=\log _{e} x$ is differentiated, and the curve $\frac{d y}{d x}=\frac{1}{x}$ is integrated graphically, and the area of portions of the rectangular hyperbola between given ordinates found.

The above is a brief outline of the nature and scope of the Calculus work of our third year course, and in conclusion I would repeat that the work is of the simplest kind, and does not require much skill in algebraic manipulation, the object aimed at being to give the boys, before leaving school, a start in the subject, with an appreciation of its value, so that they will continue the study at evening classes.

I would like to add that it is now seven years since we first introduced this subject in the school. During this time the course has been modified, and many new methods tried, and I am glad that your committee has given me the opportunity to bring it before you.

In spite of the criticism which may be urged against this method or that method, I hope that it will be felt generally that this branch of mathematics ought to find a place in the curriculum of every secondary school.

W. KNowles. 